OPEN ACCESS

Edited by:

Marilia Seelaender

University of São Paulo, Brazil

Reviewed by:

Maryam Ebadi,

University of Alberta, Canada

Kazuto Tajiri,

University of Toyama University

Hospital, Japan

Vera C. Mazurak,

University of Alberta, Canada

*Correspondence:

Daniel-Corneliu Leucuta

dleucuta@umfcluj.ro

Specialty section:

This article was submitted to

Clinical Nutrition,

a section of the journal

Frontiers in Nutrition

Received: 30 July 2021 Accepted: 03 January 2022 Published: 27 January 2022

Citation:

Ismaiel A, Bucsa C, Farcas A,

Leucuta D-C, Popa S-L and Dumitrascu DL (2022) Effects of Branched-Chain Amino Acids on Parameters Evaluating Sarcopenia in Liver Cirrhosis: Systematic Review and Meta-Analysis. Front. Nutr. 9:749969. doi: 10.3389/fnut.2022.749969

\section{Effects of Branched-Chain Amino Acids on Parameters Evaluating Sarcopenia in Liver Cirrhosis: Systematic Review and Meta-Analysis}

\author{
Abdulrahman Ismaiel ${ }^{1}$, Camelia Bucsa ${ }^{2}$, Andreea Farcas ${ }^{2}$, Daniel-Corneliu Leucuta ${ }^{3 *}$, \\ Stefan-Lucian Popa ${ }^{1}$ and Dan L. Dumitrascu ${ }^{1}$
}

1 2nd Department of Internal Medicine, "Iuliu Hatieganu" University of Medicine and Pharmacy, Cluj-Napoca, Romania, ${ }^{2}$ Drug Information Research Center, "Iuliu Hatieganu" University of Medicine and Pharmacy, Cluj-Napoca, Romania, ${ }^{3}$ Department of Medical Informatics and Biostatistics, "Iuliu Hatieganu" University of Medicine and Pharmacy, Cluj-Napoca, Romania

Introduction: Sarcopenia is a major element of malnutrition in liver cirrhosis (LC) and is present in 30-70\% of this population, being associated with a poor overall prognosis due to related complications such as hepatic encephalopathy, ascites, and portal hypertension. This systematic review and meta-analysis aimed to evaluate the effects of branched-chain amino acids (BCAA) supplementation on several parameters used to assess sarcopenia in LC.

Materials and Methods: A comprehensive systematic electronic search was performed in PubMed, EMBASE, Scopus, Cochrane Library, and ClinicalTrials.gov databases using predefined keywords. We included full articles that satisfied the inclusion and exclusion criteria. Quality assessment of included studies was conducted using Cochrane Collaboration's tool and NHLBI quality assessment tools for interventional and observational studies, respectively. The principal summary outcome was the mean difference (MD) in the evaluated parameters. We performed a pre- and post-intervention analysis and comparison between two intervention groups (BCAA vs. controls) of the evaluated parameters when applicable.

Results: A total of 12 studies involving 1,225 subjects were included in our qualitative synthesis and five in our quantitative synthesis. At baseline vs. post-intervention assessment, subjects receiving BCAA supplementation were found to have a significant improvement in skeletal muscle index (SMI) $(-0.347$ [95\% Cl -0.628-0.067; $p$-value 0.015]) and mid-arm muscle circumference (MAMC) $(-1.273$ [95\% Cl (-2.251-0.294; $p$-value 0.011]). However, no improvements were reported in handgrip (-0.616 [95\% Cl $-2.818-1.586 ; p$-value 0.584]) and triceps subcutaneous fat (1.10 [95\% Cl -0.814-3.014; $p$-value 0.263]).

Conclusion: Following BCAA supplementation, several parameters used to evaluate sarcopenia in LC patients were found to be improved, including SMI and MAMC. Nevertheless, no improvements were seen in handgrip and triceps subcutaneous fat. 
Results should be interpreted with caution due to the limited methodological quality of the included studies.

Keywords: branched-chain amino acids (BCAA), sarcopenia, liver cirrhosis, anthropometric parameters, skeletal muscle index (SMI), mid-arm muscle circumference (MAMC), systematic review, meta-analysis

\section{INTRODUCTION}

Sarcopenia is a syndrome proposed by Rosenberg in 1989, defined as an age-related muscle mass reduction and abnormalities in muscle function, including muscle strength and physical performance $(1,2)$. Sarcopenia can be categorized as primary when associated with aging or secondary when related to an underlying condition such as systemic diseases, including chronic liver disease (CLD), being one of the main causes of secondary sarcopenia $(3,4)$.

Sarcopenia is a major element of malnutrition in liver cirrhosis and has been reported to be prevalent in 30$70 \%$ of this population (5). Several causes lead to the development of sarcopenia in patients with cirrhosis. These include malabsorption, dysregulated metabolism, reduced nutritional intake, hormonal alterations, increased loss of muscle, and hyperammonemia $(6,7)$. The overall prognosis in patients with cirrhosis is affected by sarcopenia due to other related complications such as hepatic encephalopathy, ascites, and portal hypertension $(8,9)$. The importance of sarcopenia is that it is associated with reduced quality of life, survival, mobility, and cardiopulmonary performance, as well as unfavorable metabolic outcomes and increased infection rates when compared to non-sarcopenic patients $(3,4)$.

Most studies evaluating sarcopenia were conducted in community-dwelling elderly patients for whom several consensus definitions have been published $(3,4,10)$. Nevertheless, applying the existing consensus definition in liver cirrhosis patients is challenging due to muscle mass changes that develop in this population influencing the measurements, possibly due to altered hepatic function and water retention in peripheral edema and ascites. Furthermore, a clear consensus defining sarcopenia in liver cirrhosis patients remains required. Several tests have been used to assess sarcopenia in cirrhosis (11). Figure 1 summarizes the frequently used tests to evaluate sarcopenia, including muscle mass, function, and strength evaluation (11).

Although the clinical significance of sarcopenia in cirrhosis has been widely recognized, effective therapies are still to be discovered, mainly due to the limited available data describing the mechanisms relating sarcopenia to cirrhosis, a condition believed to be associated with a state of anabolic resistance $(12,13)$. Studied therapeutic approaches include diets rich in protein and fiber, nutrients supplementation with branchedchain amino acids (BCAAs), minerals, and vitamins, as well as exercise (14-16).

The concentration of BCAAs in plasma and skeletal muscle are reduced in cirrhosis $(17,18)$. BCAAs have been shown to be helpful as a nutritional supplement in liver cirrhosis (19-21). Several clinical trials reported the efficacy of BCAAs for nutritional status, general status, hepatic encephalopathy, and quality of life (22-25). Therefore, it is expected that BCAA supplementation may be considered a useful therapeutic modality in treating decreased muscle mass and strength that accompany secondary sarcopenia.

This comprehensive systematic review and meta-analysis aimed to investigate the effects of BCAA supplementation in observational and interventional studies on several parameters used to assess sarcopenia in patients with liver cirrhosis, including muscle mass, function, and strength evaluation such as mid-arm muscle circumference (MAMC) and skeletal muscle index (SMI), as well as handgrip strength and triceps subcutaneous fat assessment.

\section{METHODS}

This systematic review and meta-analysis was written according to the Preferred Reporting Items for Systematic Reviews and Meta-Analyses (PRISMA) checklist 2020 (26).

\section{Data Sources and Search Strategy}

We aimed to review the currently available evidence published on PubMed, EMBASE, Scopus, Cochrane Library, and ClinicalTrials.gov, trying to answer the PICOS research question: identifying the population of patients with liver cirrhosis, with a BCAA supplementation intervention, vs. a comparator (maltodextrin), or a before after design, to observe several parameters assessing sarcopenia, as outcomes, including SMI and MAMC, as well as handgrip strength and triceps subcutaneous fat, in interventional or observational studies. A description of the conducted search strategy is provided in Supplementary Material 1. Moreover, we performed a manual search for missed publications by screening the references of included articles to minimize the risk of missing relevant studies. The conducted search included published articles from inception up to 14 July 2021. No search filters or restrictions were applied in regards to duration, country, or language. Afterward, a screening assessment was conducted by evaluating titles and abstracts for appropriateness. Articles that were selected based on the inclusion and exclusion criteria were evaluated through a full-text review. Eligibility of the evaluated studies was performed independently by two authors (A.I. and D.C.L.), and data extraction from eligible studies was performed by two authors (C.B. and A.F.), while resolving any discrepancies by mutual consensus.

\section{Eligibility Criteria}

Inclusion criteria of original articles were as follows: (1) Full article interventional (clinical trials, RCTs) or observational studies (observational cohort population-based/ hospitalbased, cross-sectional or case-control designs), evaluating 


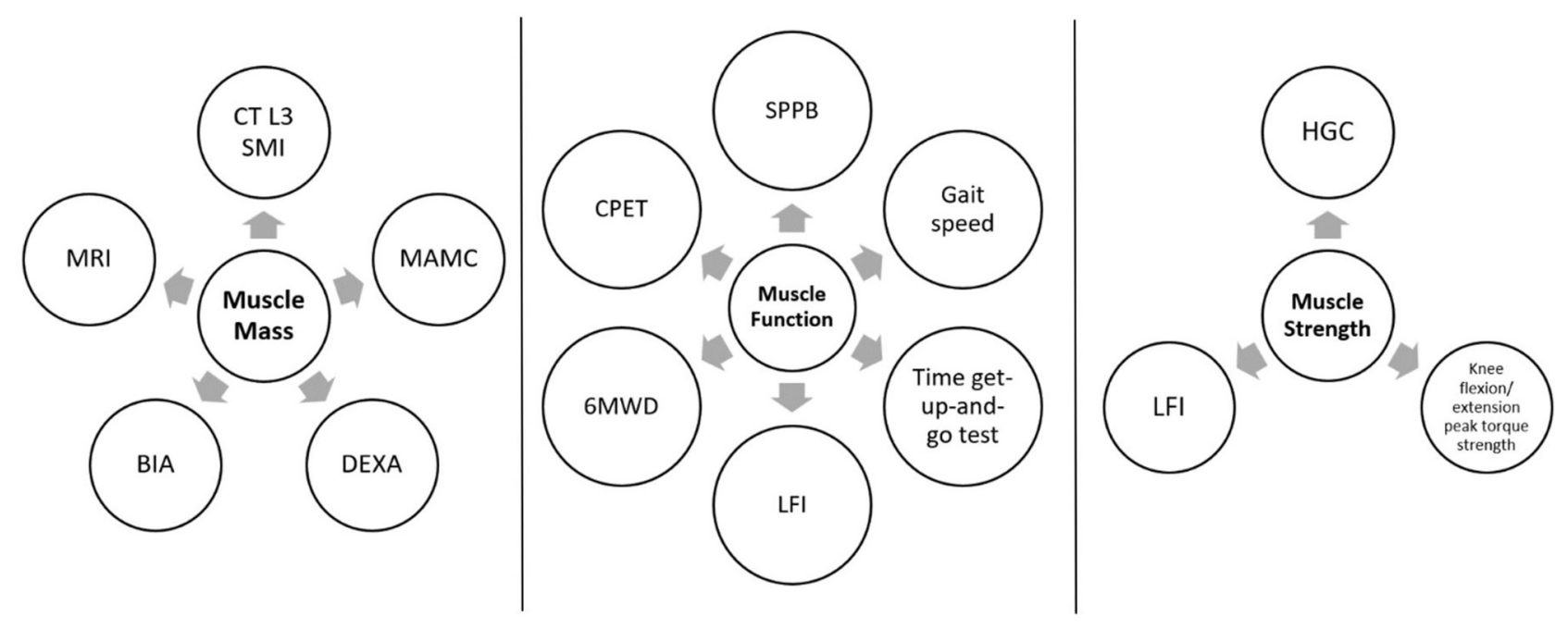

FIGURE 1 | Tests used to evaluate sarcopenia, assessing muscle mass, function, and strength. CT, computed tomography; SMI, skeletal muscle index; MRI, magnetic resonance imaging; MAMC, mid-arm muscle circumference; DEXA, dual-energy X-ray absorptiometry; BIA, bioimpedance analysis; SPPB, short physical performance battery test; LFI, Liver Frailty Index; 6MWD, 6-minute walk distance; CPET, cardiopulmonary exercise testing; HGC, Handgrip strength.

the effects of BCAA supplementation on anthropometric and functional parameters assessing sarcopenia in patients with cirrhosis; (2) Liver cirrhosis evaluated using liver biopsy or imaging techniques such as ultrasonography, computed tomography (CT), magnetic resonance imaging (MRI), codes such as International Classification of Diseases (ICD), or as per study definition; (3) Parameters assessing sarcopenia according to each studies definition; (4) Human studies only; and (5) Studies published in English, French, German or Romanian languages.

Exclusion criteria were as follows: (1) Subjects with endstage liver disease who received a liver transplant; (2) Presence of hepatocellular carcinoma; (3) Editorials, letters to the editor, case reports, conference abstracts, literature, and systematic reviews, practice guidelines, commentaries, clinical trial registrations, abstracts published without a full-text article; and (4) Experimental studies.

\section{Risk of Bias Assessment in Individual Studies}

The risk of bias for randomized controlled trials was assessed using the Cochrane Collaboration's tool (27). The quality assessment was based on randomized sequence generation, treatment allocation concealment, blinding, and completeness of the outcome data, in addition to selective outcome reporting and other sources of bias.

For non-RCT studies, we used the National Heart, Lung, and Blood Institute (NHLBI) quality assessment tools (28). Two NHLBI tools were used, the Quality Assessment Tool for Observational Cohort and Cross-Sectional Studies, as well as the Quality Assessment Tool for Before-After (Pre-Post) Studies with No Control Group.
We used these evaluation tools to evaluate bias risk and internal validity in individual studies in a similar manner. The risk of bias in individual studies was evaluated by two authors independently (A.I. and D.C.L.). In case of disagreement, a consensus was reached through a discussion.

\section{Summary Measures and Synthesis of Results}

The principal summary outcome was the mean difference (MD) of several parameters, including SMI, MAMC, handgrip, and triceps subcutaneous fat. For the summary outcomes, we computed the estimates of the random effects using restricted maximum likelihood to estimate the heterogeneity variance, since we assumed clinical variability between the studies. We conducted the data analyses of the meta-analysis using $\mathrm{R}$ with Metafor package (OpenMeta [Analyst]) $(29,30)$. Between-study heterogeneity was evaluated using the $\chi^{2}$ based Q-test and $I^{2}$. According to the recommendations of the Cochrane Handbook (31) for identifying and measuring heterogeneity, we estimated $I^{2}$ values of $0 \%$ to $40 \%$ as not important; $30 \%$ to $60 \%$ as moderate heterogeneity; $50-90 \%$ as substantial heterogeneity; and $75 \%$ to $100 \%$ as considerable heterogeneity.

The estimated total effect size analysis was calculated using the random-effects model and MD. In studies that reported medians and interquartile ranges, we calculated the mean and standard deviation (SD) to perform statistical analyses of the obtained data (32). In studies reporting results at baseline and postintervention data, the mean change and SD change were used if they were reported. Still, in case they were not reported, they were calculated based on the before and after values according to the Cochrane Handbook recommendation using the correlation coefficient from the same study or imputed from a similar study (27). Data was reported from each study as the estimated MD 
with a $95 \%$ confidence interval (CI). A statistically significant $p$ value was considered when $<0.05$. The analyses were conducted if two or more studies evaluated similar groups and reported the same outcome using mean $+/-\mathrm{SD}$ or median (IQR). We also performed baseline and post-intervention analysis when available in single studies. For baseline and post-intervention analysis, we only included groups that received solely BCAA supplementation. We were not able to perform publication bias assessment due to the limited number of included studies.

\section{RESULTS}

\section{General Results}

Figure 2 outlines the PRISMA flow diagram describing the performed search strategy. The initial search yielded 191 articles (PubMed $n=29$, EMBASE $n=70$, Scopus $n=56$ articles, ClinicalTrials.gov $n=23$ articles, Cochrane Library $n=13$ articles). A total of 63 studies were removed after being detected as duplicates. After excluding the duplicates, 128 articles underwent a preliminary screening by assessing the title and abstract for inclusion and exclusion criteria fulfillment. During the screening phase we excluded 110 articles. Eighteen articles were sought for retrieval, out of which the full text of one article was not found (we contacted the authors by email, but we didn't receive any feedback). We performed a thorough reading and evaluation of the full texts for further eligibility assessment of the remaining 17 articles. We excluded six out of these articles with reasons as follows: (1) no clear BCAA group $(33,34),(2)$ abstract without full text (35), (3) involving hepatocellular carcinoma (HCC) patients (36), (4) not involving cirrhosis patients (37), and (5) outcome influenced selection (38). Accordingly, the qualitative synthesis included 11 articles, out of which five were included in the quantitative synthesis (38-49).

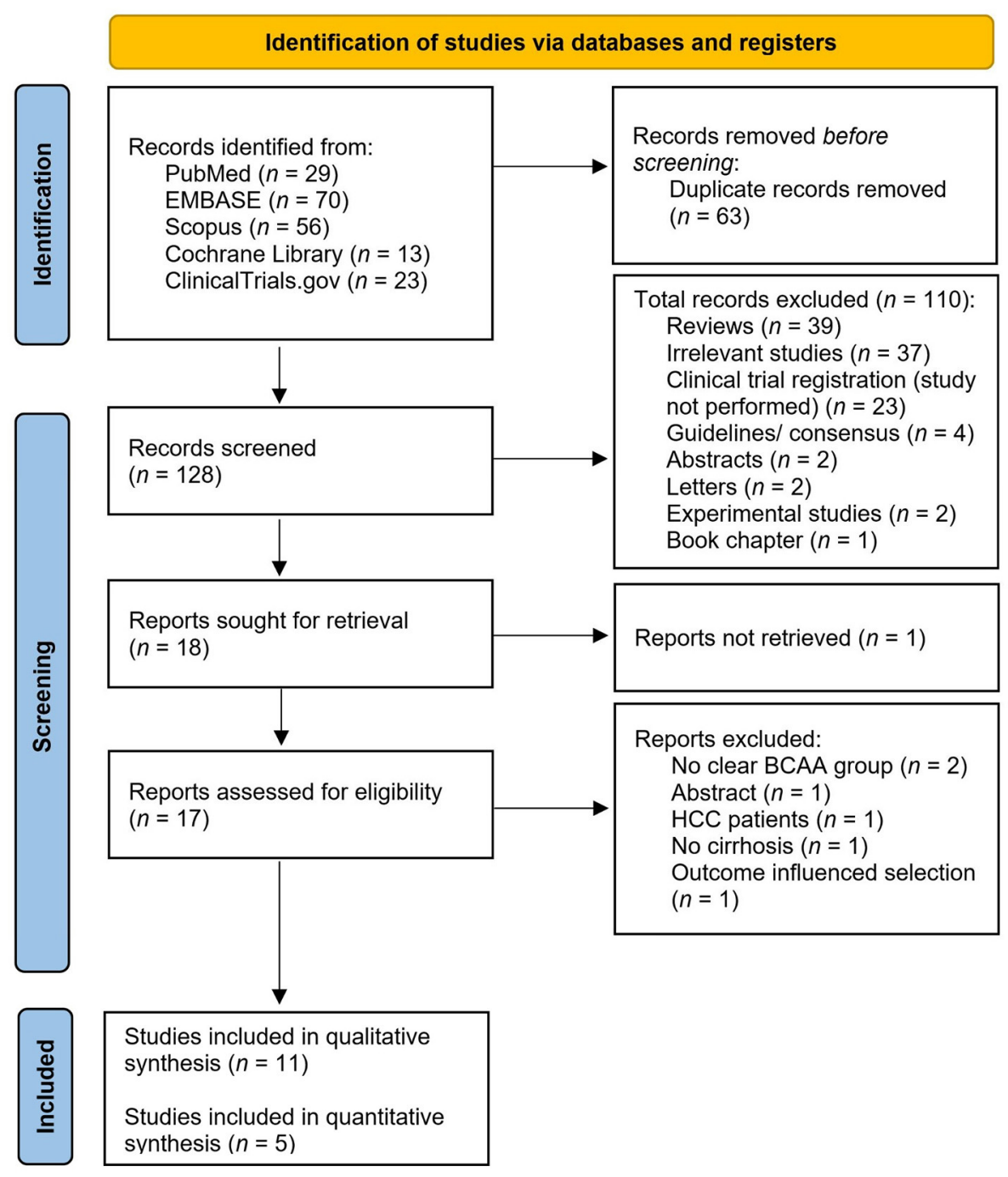

FIGURE 2 | PRISMA flow diagram describing the identification, screening, and inclusion phases. 


\section{Study Characteristics}

The main characteristics of included studies are summarized in Table 1. This systematic review and meta-analysis included a total number of 1,215 individuals (394 individuals in RCTs, 821 individuals in observational studies).

Four studies had an interventional study design (39, 40, 46, 49), five studies had a prospective study design $(42-45,47)$, and two had a retrospective design $(41,48)$. Seven studies were undertaken in Asia (Japan $n=7$ ), two in Europe (multicenter $n=1$, Spain $n=1$ ), and two in the Americas (USA $n=1$, Mexico $n=1)$.

\section{Effects of BCAA Supplementation on Sarcopenia in Patients With Cirrhosis}

Several parameters were evaluated in the included studies, assessing the effects of BCAA supplementation in sarcopenic patients with liver cirrhosis, including muscle mass, function, and strength, as demonstrated in Supplementary Table 1. These parameters included MAMC, SMI, skeletal muscle area, handgrip strength, tricipital skinfold thickness, bicipital skinfold thickness, suprailiac skinfold thickness, subscapular skinfold thickness, midarm muscle area, midarm fat area, fat mass, fat-free mass.

\section{Skeletal Muscle Index Improvement (Baseline and Post-intervention)}

A total of two studies reported mean $+/-$ SD or median (IQR) for the SMI $\left(\mathrm{cm}^{2} / \mathrm{m}^{2}\right)$ involving baseline values and post-BCAA supplementation $(45,49)$. Figure 3 summarizes the obtained results regarding SMI, which was evaluated using CT scan and bioelectrical impedance analysis.

The pooled analysis assessing the SMI in baseline minus post-BCAA supplementation groups demonstrated that SMI significantly increased after the BCAA supplementation, the difference being of -0.347 (95\% CI $-0.628-0.067)$, $p$-value of 0.015 . Very low heterogeneity was reported with an $I^{2}=0 \%$ and p-value 0.589 .

\section{Mid-Arm Muscle Circumference Post-intervention (BCAA vs. M-DXT)}

A total of two studies reported mean $+/-$ SD for the MAMC $(\mathrm{cm})$ comparing BCAA group vs. maltodextrins (M-DXT) group (39, 40). Figure 4 summarizes the obtained results regarding MAMC.

In the pooled analysis assessing the MAMC in BCAA group vs. M-DXT group, we observed overall larger MAMC post-intervention values in the M-DXT group compared to BCAA group, but they did not reach the statistically significant threshold, the mean difference between the groups being-0.443 (95\% CI -0.994-0.240), $p$-value of 0.116 . Substantial heterogeneity was reported with an $I^{2}=$ $75.63 \%$ and $p$-value 0.043 .

\section{Mid-Arm Muscle Circumference Improvement (Baseline and Post-intervention)}

A total of two studies reported mean $+/-$ SD for the MAMC $(\mathrm{cm})$ comparing baseline values and post-BCAA supplementation (40, 46). Figure 5 summarizes the obtained results regarding MAMC.
The pooled analysis assessing the difference in MAMC values between baseline and post-BCAA supplementation groups demonstrated that MAMC values significantly increased after the BCAA supplementation, the MD being of -1.273 (95\% CI $-2.251-0.294), p$-value of 0.011 . Substantial heterogeneity was reported with an $I^{2}=90.98 \%$ and $p$-value $<0.001$.

\section{Handgrip Change (Baseline and Post-intervention)}

A total of two studies reported mean $+/-$ SD or median (IQR) for handgrip $(\mathrm{kg})$ comparing baseline values and post-BCAA supplementation $(40,49)$. Figure 6 summarizes the obtained results regarding handgrip.

In the pooled analysis assessing the difference in handgrip between baseline and post-BCAA supplementation we observed an increase in handgrip, albeit non-statistically significant, with an overall MD of -0.616 (95\% CI $-2.818-1.586)$, $p$-value of 0.584 . Substantial heterogeneity was reported with an $I^{2}=$ $59.29 \%$ and $p$-value 0.117 .

Handgrip was evaluated in one individual study separately, comparing BCAA group vs. DXT group, assessing baseline vs. post-intervention difference. We observed a larger increase in handgrip (baseline vs. post-intervention) in BCAA group compared to DXT group, albeit non-statistically significant, with an MD of -1.0 (95\% CI -2.674-0.674), p-value of 0.244 .

\section{Triceps Subcutaneous Fat Change (Baseline and Post-intervention)}

Triceps subcutaneous fat was evaluated in one individual study separately, comparing BCAA group vs. controls, assessing baseline vs. post-intervention difference. We observed a nonstatistically significant decrease in triceps subcutaneous fat (baseline vs. post-intervention) in BCAA group compared to controls, with an MD of 1.10 (95\% CI -0.814-3.014), $p$-value of 0.263 .

\section{Quality Assessment}

Four articles were evaluated using the Cochrane Collaboration's tool $(39,40,46,49)$, five articles using the NHLBI Quality Assessment Tool for Observational Cohort and Cross-Sectional Studies $(38,41-43,48)$, and three articles using the NHLBI Quality Assessment Tool for Before-After (Pre-Post) Studies with No Control Group $(44,45,47)$, as demonstrated in Supplementary Tables 2-4.

Several issues were reported regarding bias in the assessed articles. Regarding RCTs evaluated in our review, although all four included RCTs had a low risk for selection bias related to random sequence generation, two of them presented an unclear risk of allocation concealment bias $(46,49)$. Moreover, one article presented a high risk of performance bias related to blinding of the participants and personnel as well as detection bias evaluated as outcome assessment blinding (46), while another was evaluated as unclear for both parameters being assessed (49). Incomplete outcome data, considered as attrition bias, was high in two included RCTs $(40,46)$. All RCTs included in this review had an unclear risk of bias regarding reporting bias and other possible sources of bias $(39,40,46,49)$. 
TABLE 1 | Characteristics of studies included in the systematic review and meta-analysis.

\begin{tabular}{|c|c|c|c|c|c|c|c|c|c|}
\hline No. & Study, setting & Study type & $\begin{array}{l}\text { Population } \\
\text { included }\end{array}$ & $\begin{array}{l}\text { BCAA/BCAA + } \\
\text { Intervention }\end{array}$ & Comparison & $\begin{array}{l}\text { Number of } \\
\text { patients } \\
\text { included }\end{array}$ & $\begin{array}{l}\text { Study } \\
\text { participant's } \\
\text { characteristics }\end{array}$ & Measurements & Main findings \\
\hline 1 & $\begin{array}{l}\text { Marchesini et al., } \\
\text { 2003; Setting: } \\
\text { Europe }\end{array}$ & $\begin{array}{l}\text { RCT, } \\
\text { double-blind, } \\
\text { multicenter }\end{array}$ & $\begin{array}{l}\text { Patients with liver } \\
\text { cirrhosis, portal } \\
\text { hypertension, with } \\
\text { Child-Pugh score } \\
\geq 7\end{array}$ & $\begin{array}{l}\text { BCAA ( } 14.4 \mathrm{~g} / \text { day } \\
\text { composed of } 1.2 \mathrm{~g} \\
\text { L-leucine, } 0.6 \mathrm{~g} \\
\text { L-isoleucine, and } 0.6 \mathrm{~g} \\
\text { L-valine, } 225 \mathrm{Kcal} / \text { day) } \\
\text { for } 12 \text { months }\end{array}$ & $\begin{array}{l}\text { Lactoalbumin OR } \\
\text { Maltodextrins for } \\
12 \text { months }\end{array}$ & $\begin{array}{l}n=59 \text { BCAA } \\
\text { group/ } 56 \\
\text { Lactoalbumin } \\
\text { group } / n=59 \\
\text { Maltodextrins } \\
\text { group }\end{array}$ & $\begin{array}{l}59 \mathrm{Y} \text { in BCAA } \\
\text { group/60 years in } \\
\text { Lactoalbumin } \\
\text { group/59 } \mathrm{Y} \text { in } \\
\text { Maltodextrins } \\
\text { group; } 36.8 \% \mathrm{~F}\end{array}$ & $\begin{array}{l}\text { Midarm } \\
\text { circumference (to } \\
\text { the nearest } \\
\text { millimeter) using a } \\
\text { tape meter; } \\
\text { skinfold thickness } \\
\text { using a caliper }\end{array}$ & $\begin{array}{l}\text { Significant } \uparrow \text { in } \\
\text { triceps skinfold } \\
\text { thickness and } \\
\text { midarm fat area in } \\
\text { BCAA group }\end{array}$ \\
\hline 2 & $\begin{array}{l}\text { Les et al., 2011; } \\
\text { Setting: Spain }\end{array}$ & $\begin{array}{l}\text { RCT, } \\
\text { double-blind, } \\
\text { multicenter }\end{array}$ & $\begin{array}{l}18-85 \text { years, with } \\
\text { liver cirrhosis and } \\
\text { hospitalized for } \\
\text { hepatic } \\
\text { encephalopathy, } \\
\text { compliant with a } \\
\text { standard diet } 2 \\
\text { weeks before } \\
\text { inclusion }\end{array}$ & $\begin{array}{l}\text { BCAA ( } 30 \mathrm{~g} / \text { day, } 120 \\
\text { Kcal/day) composed of } \\
\text { leucine: } 13.5 \mathrm{~g} \text {, } \\
\text { isoleucine: } 9 \mathrm{~g} \text {, valine: } \\
7.5 \mathrm{~g} \text { or MDX, for } 56 \\
\text { weeks }\end{array}$ & Maltodextrin & $\begin{array}{l}n=58 \mathrm{BCAA} \\
\text { group } / n=58 \\
\text { Maltodextrin group }\end{array}$ & $\begin{array}{l}64.1 \mathrm{Y} \text { in BCAA } \\
\text { group/ } 62.5 \mathrm{Y} \text { in } \\
\text { Maltodextrin } \\
\text { group; } 24 \% \mathrm{~F}\end{array}$ & $\begin{array}{l}\text { Midarm muscle } \\
\text { circumference; } \\
\text { muscular strength } \\
\text { by handgrip } \\
\text { (methods of } \\
\text { measurements not } \\
\text { provided) }\end{array}$ & $\begin{array}{l}\text { Midarm muscle } \\
\text { circumference and } \\
\text { handgrip } \uparrow \text { in } \\
\text { patients in the } \\
\text { BCAA group }\end{array}$ \\
\hline 3 & $\begin{array}{l}\text { Hanai et al., 2015; } \\
\text { Setting: Japan }\end{array}$ & $\begin{array}{l}\text { Retrospective } \\
\text { cohort study }\end{array}$ & $\begin{array}{l}>18 \text { years with } \\
\text { liver cirrhosis }\end{array}$ & $\begin{array}{l}\text { aBCAA } 12 \mathrm{~g} / \text { day, } 4 \mathrm{~g} \text { of } \\
\text { BCAA per sachet } \\
\text { composed of } 952 \mathrm{mg} \\
\text { L-isoleucine, } 1904 \mathrm{mg} \\
\text { L-leucine, and } 1144 \mathrm{mg} \\
\text { L-valine, for }>12 \\
\text { months }\end{array}$ & NA & $\begin{array}{l}n=94 \text { BCAA } \\
\text { group } / n=36 \\
\text { non-BCAA group }\end{array}$ & $\begin{array}{l}66 \mathrm{Y} \text { in BCAA } \\
\text { group/64 } \mathrm{Y} \text { in } \\
\text { non-BCAA group; } \\
42 \% \mathrm{~F}\end{array}$ & SMl using CT scan & $\begin{array}{l}\text { A possible } \\
\text { association } \\
\text { between BCAA } \\
\text { administration and } \\
\uparrow \text { outcome in four } \\
\text { sarcopenic } \\
\text { patients with liver } \\
\text { cirrhosis was } \\
\text { observed }\end{array}$ \\
\hline 4 & $\begin{array}{l}\text { Tsien et al., 2015; } \\
\text { Setting: Cleveland, } \\
\text { USA }\end{array}$ & $\begin{array}{l}\text { Prospective } \\
\text { study }\end{array}$ & $\begin{array}{l}\text { Patients with } \\
\text { alcoholic cirrhosis, } \\
\text { with Child-Pugh } \\
\text { score } \leq 7 \text { and } \\
\text { healthy controls }\end{array}$ & $\begin{array}{l}\text { Leucine enriched } \\
\text { BCAA, } 15 \mathrm{~g} \text { composed } \\
\text { of BCAA/LEU }(7.5 \mathrm{~g} \\
\text { L-leucine, } 3.75 \mathrm{~g} \\
\text { L-isoleucine, } 3.75 \mathrm{~g} \\
\text { L-valine), single } \\
\text { administration in } \\
\text { patients with cirrhosis }\end{array}$ & $\begin{array}{l}\text { Leucine enriched } \\
\text { BCAA, } 15 \mathrm{~g} \text { single } \\
\text { administration in } \\
\text { healthy controls }\end{array}$ & $\begin{array}{l}n=6 \text { patients with } \\
\text { cirrhosis } / n=8 \\
\text { healthy controls }\end{array}$ & $\begin{array}{l}54 \mathrm{Y} \text { in patients } \\
\text { with cirrhosis } \\
\text { group } / 45 \mathrm{Y} \text { in } \\
\text { healthy controls; } \\
35.7 \% \mathrm{~F}\end{array}$ & $\begin{array}{l}\text { Body composition } \\
\text { characteristics } \\
\text { assessed using } \\
\text { dual-energy Xray } \\
\text { absorptiometry. } \\
\text { Muscle expression } \\
\text { of myostatin, } \\
\text { mTOR targets, } \\
\text { autophagy } \\
\text { markers, protein } \\
\text { ubiquitination and } \\
\text { intracellular amino } \\
\text { acid deficiency } \\
\text { sensor by muscle } \\
\text { biopsy }\end{array}$ & $\begin{array}{l}\text { Impaired mTOR1 } \\
\text { signaling and } \\
\uparrow \text { autophagy in } \\
\text { skeletal muscle of } \\
\text { alcoholic cirrhosis } \\
\text { patients is acutely } \\
\text { reversed by } \\
\text { leucine enriched } \\
\text { BCAA }\end{array}$ \\
\hline
\end{tabular}




\begin{tabular}{|c|c|c|c|c|c|c|c|c|c|}
\hline No. & Study, setting & Study type & $\begin{array}{l}\text { Population } \\
\text { included }\end{array}$ & $\begin{array}{l}\text { BCAA/BCAA + } \\
\text { Intervention }\end{array}$ & Comparison & $\begin{array}{l}\text { Number of } \\
\text { patients } \\
\text { included }\end{array}$ & $\begin{array}{l}\text { Study } \\
\text { participant's } \\
\text { characteristics }\end{array}$ & Measurements & Main findings \\
\hline 5 & $\begin{array}{l}\text { Hiraoka et al., } \\
\text { 2017; Setting: } \\
\text { Japan }\end{array}$ & $\begin{array}{l}\text { Observational, } \\
\text { prospective } \\
\text { (pre-post } \\
\text { intervention) }\end{array}$ & $\begin{array}{l}\text { Patients with liver } \\
\text { cirrhosis }\end{array}$ & $\begin{array}{l}\text { BCAA (protein } 13.5 \mathrm{~g} \text {, } \\
210 \mathrm{kcal} / \text { day including } \\
\text { L-leucine } 1922.5 \mathrm{mg} \text { ) + } \\
\text { walking exercise } \\
\text { (additional 2,000 steps } \\
\text { daily) }\end{array}$ & NA & $n=33$ (one group) & 67 Y; 60.6\% F & $\begin{array}{l}\text { Muscle volume } \\
\text { using bioelectrical } \\
\text { impedance; } \\
\text { handgrip strength } \\
\text { using a hand } \\
\text { dynamometer; leg } \\
\text { strength using a } \\
\text { position } \\
\text { controllable cycle } \\
\text { ergometer }\end{array}$ & $\begin{array}{l}\text { BCAA } \\
\text { supplementation } \\
\text { and walking } \\
\text { exercise were } \\
\text { found to be } \\
\text { effective and easily } \\
\text { implemented for } \\
\text { 个muscle volume } \\
\text { and strength in } \\
\text { liver cirrhosis } \\
\text { patients }\end{array}$ \\
\hline 6 & $\begin{array}{l}\text { Uojima et al., } \\
\text { 2017; Setting: } \\
\text { Japan }\end{array}$ & $\begin{array}{l}\text { Single-center, } \\
\text { prospective } \\
\text { study } \\
\text { (pre-post } \\
\text { intervention) }\end{array}$ & $\begin{array}{l}\text { Patients }>20 \\
\text { years, with liver } \\
\text { cirrhosis, with } \\
\text { albumin level }<3.5 \\
\mathrm{~g} / \mathrm{dl} \text { after standard } \\
\text { nutrition therapy } \\
\text { for at least } 28 \text { days }\end{array}$ & $\begin{array}{l}\text { BCAA (2*50 g/day, } 420 \\
\text { Kcal/day), one package } \\
\text { of BCAA }(50 \mathrm{~g}) \text { was } \\
\text { composed of } 13.5 \mathrm{~g} \text { of } \\
\text { protein, including } \\
\text { L-leucine, L-isoleucine, } \\
\text { and L-valine, which } \\
\text { provided } 210 \mathrm{kcal} \text { of } \\
\text { energy; for } 24 \text { weeks }\end{array}$ & NA & $n=82$ (one group) & 69 Y; 44\% F & $\begin{array}{l}\text { SMI using } \\
\text { bioelectrical } \\
\text { impedance } \\
\text { analysis; hand grip } \\
\text { using a grip } \\
\text { dynamometer }\end{array}$ & $\begin{array}{l}\text { BCAA } \\
\text { supplementation } \\
\uparrow \text { low muscle } \\
\text { strength in } \\
\text { patients with } \\
\text { chronic liver } \\
\text { disease, but did } \\
\text { not increase } \\
\text { muscle mass } \\
\text { during the } \\
\text { treatment period }\end{array}$ \\
\hline 7 & $\begin{array}{l}\text { Kitajima et al., } \\
\text { 2017; Setting: } \\
\text { Japan }\end{array}$ & $\begin{array}{l}\text { Observational, } \\
\text { prospective; } \\
\text { (pre-post } \\
\text { intervention) }\end{array}$ & $\begin{array}{l}\text { Patients with liver } \\
\text { cirrhosis }\end{array}$ & $\begin{array}{l}\text { BCAA granules (25-35 } \\
\text { Kcal/kg/day and } \\
\text { protein intake to } \\
1.0-1.4 \mathrm{~kg} / \text { day), each } \\
\text { packet of BCAA } \\
\text { contained } 952 \mathrm{mg} \\
\text { L-isoleucine, } 1904 \mathrm{mg} \\
\text { L-leucine, and } 1144 \mathrm{mg} \\
\text { L-valine; for } 48 \text { weeks }\end{array}$ & NA & $n=21$ (one group) & 71.3 Y; $57.1 \%$ F & $\begin{array}{l}\text { Skeletal muscle } \\
\text { volume using CT } \\
\text { scan and } \\
\text { bioelectrical } \\
\text { impedance } \\
\text { analysis; } \\
\text { intramuscular } \\
\text { adipose tissue } \\
\text { content using CT } \\
\text { scan }\end{array}$ & $\begin{array}{l}\text { BCAA were } \\
\text { associated with } \\
\text { } a \text { albumin levels in } \\
\text { patients } \\
\text { with hypoalbuminemia } \\
\text { and were related } \\
\text { to maintained } \\
\text { skeletal } \\
\text { muscle mass }\end{array}$ \\
\hline 8 & $\begin{array}{l}\text { Ruiz-Margáin et } \\
\text { al., 2018; Setting: } \\
\text { Mexico }\end{array}$ & $\begin{array}{l}\text { RCT, } \\
\text { open-label }\end{array}$ & $\begin{array}{l}\text { Patients 18-65 } \\
\text { years, with liver } \\
\text { cirrhosis }\end{array}$ & $\begin{array}{l}\text { BCAA }(110 \mathrm{~g} / \text { day })+ \\
\text { High-protein, High-fiber } \\
\text { diet, composed of } \\
3.38 \mathrm{~g} \text { of L-leucine, } \\
2.75 \mathrm{~g} \text { of L-isoleucine, } \\
\text { and } 2.5 \mathrm{~g} \text { of L-valine, } \\
\text { totaling } 500 \text { Kcal for } 6 \\
\text { months }\end{array}$ & $\begin{array}{l}\text { High-protein, } \\
\text { High-fiber diet }\end{array}$ & $\begin{array}{l}n=37 \text { BCAA } \\
\text { group } / n=35 \\
\text { control group }\end{array}$ & $\begin{array}{l}54.9 \mathrm{Y} \text { in BCAA } \\
\text { group/47.8 years } \\
\text { in Control group; } \\
80.6 \% \mathrm{~F}\end{array}$ & $\begin{array}{l}\text { Triceps skinfold } \\
\text { thickness and } \\
\text { mid-arm muscle } \\
\text { circumference }\end{array}$ & $\begin{array}{l}\text { } \text { in muscle mass } \\
\text { and a decrease in } \\
\text { fat mass in the } \\
\text { BCAA group, but } \\
\text { not in the control } \\
\text { group }\end{array}$ \\
\hline
\end{tabular}


TABLE 1 | Continued

No. Study, setting

\section{Study type}

\section{Population}

included

BCAA/BCAA +

Comparison

Intervention

Number

patients
included

Study
participant's

characteristics

$9 \quad$ Hiraoka et al.,

Observational, Patients with liver Levocarnitine (1000

NA

$n=18$ (one group)

$68.4 \mathrm{Y}, 44.4 \% \mathrm{~F}$

$\mathrm{mg} /$ day) + exercise

Japan

prospective cirrhosis and

(pre-post

BCAA

(plus 2000 steps/day),

supplementation
(12.45 g/day)

for 6 months

Muscle volum

using bioelectrica

impedance

analysis; hand

(12.45 g/day)

dynamometer;

muscle strength

using position

controllable cycle

$10 \quad$ Hanai et al., 2020;

$\begin{array}{ll}\text { Observational, } & \text { Patients }>20 \\ \text { retrospective } & \text { years, with liver }\end{array}$

Setting: Japan

cohort study

cirrhosis

aBCAA (6.1 g)-enriched

powder mix daily use

composed of $1.602 \mathrm{~g}$

of L-valine, $2.037 \mathrm{~g}$ of

No administration

$n=87$ BCAA
group $/ n=436$

-leucine, and $1.923 \mathrm{~g}$

No-BCAA group

69 Y BCAA

group/66 years

No-BCAA group;

of L-isoleucine (213

$54.7 \% \mathrm{~F}$

SMI

kcal)

11 Okubo et al., $\quad$ RCT,

\begin{tabular}{|c|c|c|}
\hline $\begin{array}{l}\text { 2021; Setting: } \\
\text { Japan }\end{array}$ & open-label & $\begin{array}{l}\text { decompensated } \\
\text { cirrhosis and } \\
\text { treated with BCAA } \\
\text { for at least } 6\end{array}$ \\
\hline
\end{tabular}

Vitamin D, $2000 \mathrm{IU}$ for

12 months

No administration
$n=15$ Vitamin $\mathrm{D}$
group $/ n=17$
Control group
73 Y Vitamin D
group/70 years
Control group;
$59.4 \% \mathrm{~F}$

$\begin{array}{ll}\begin{array}{ll}\text { using a grip force } \\ \text { meter; skeletal }\end{array} & \text { group: SMl values } \\ \text { muscle volume } & \text { medificantly } \uparrow \text {; } \\ \text { using bioelectrical } & \text { rates in the SMI } \\ \text { impedance } & \text { were }+5.8 \% \text {; } \\ \text { analysis } & \text { prevalence of } \\ & \text { sarcopenia } \\ & \text { significantly } \downarrow \text { from } \\ & 80 \% \text { to } 33 \%\end{array}$

BCAA, branched-chain amino acid; SMI, skeletal muscle index; SMA, skeletal muscle area; CT, computed tomography; $F$, females; NA, not applicable; RCT, randomized-controlled trial; $Y$, years; $\uparrow$, increased; $\downarrow$, decreased; ${ }^{a}$ non-interventional retrospective studies where BCAA were previously administered according to current guideline in Japan. 
Studies

Kitajima 2017

Okubo 2021

Overall $\left(I^{\wedge} 2=0 \%, P=0.589\right)$
Estimate (95\% C.I.)

$-0.400(-0.740,-0.060)$

$-0.233(-0.733,0.266)$

$-0.347(-0.628,-0.067)$

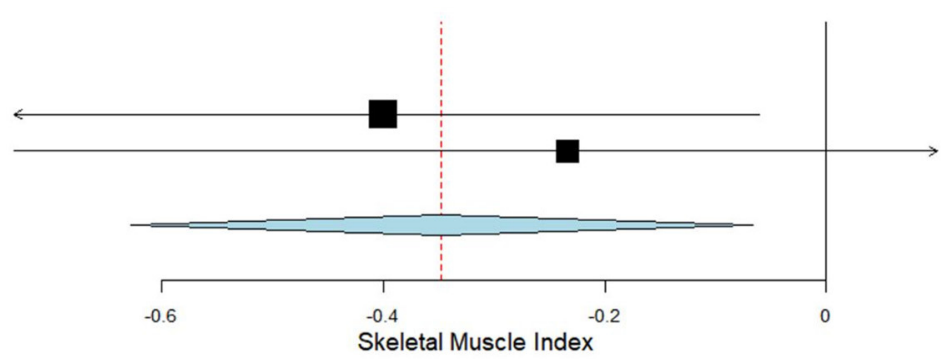

FIGURE 3 | Skeletal muscle index difference between baseline and post-BCAA supplementation groups.

Studies

Marchesini 2003

Les 2011

Overall $\left(I^{\wedge} 2=75.63 \%, P=0.043\right)$
Estimate (95\% C.I.)

$-0.720(-1.092,-0.347)$

$-0.157(-0.554,0.240)$

$-0.443(-0.994,0.109)$

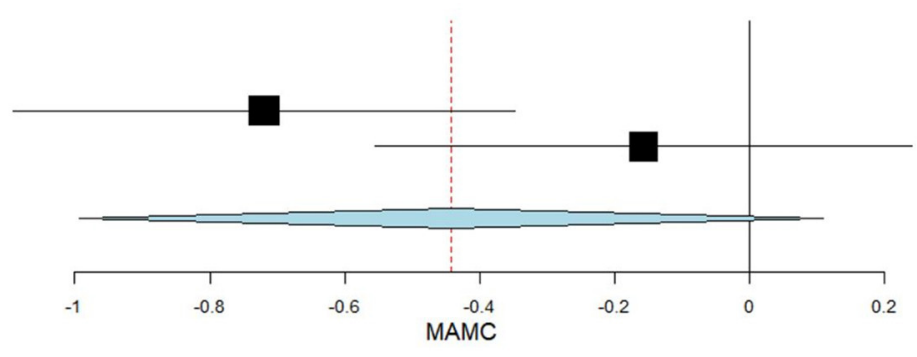

FIGURE 4 | Mid-arm muscle circumference post-intervention in BCAA group vs. M-DXT group.

Studies

A. Ruiz-Margáin 2017

Les 2011

Overall $\left(\left.\right|^{\wedge} 2=90.98 \%, P<0.001\right)$

$$
\begin{aligned}
& -1.800(-2.328,-1.272) \\
& -0.800(-1.060,-0.540)
\end{aligned}
$$

$-1.273(-2.251,-0.294)$

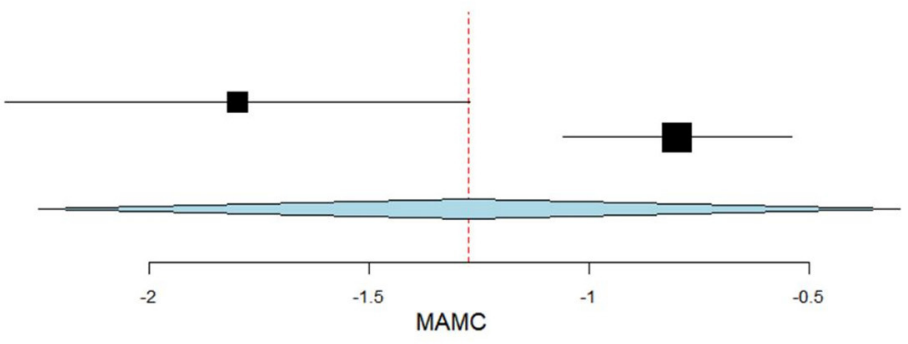

FIGURE 5 | Mid-arm muscle circumference difference between baseline and post-BCAA supplementation.

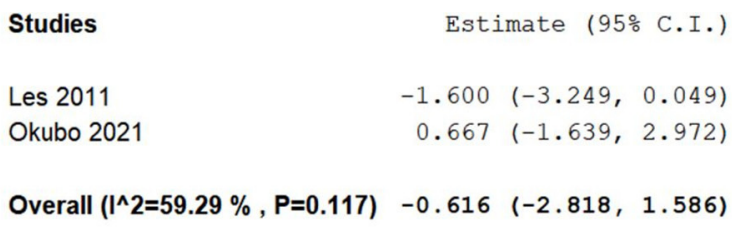

Overall $\left(I^{\wedge} 2=59.29 \%, P=0.117\right)$

$-0$

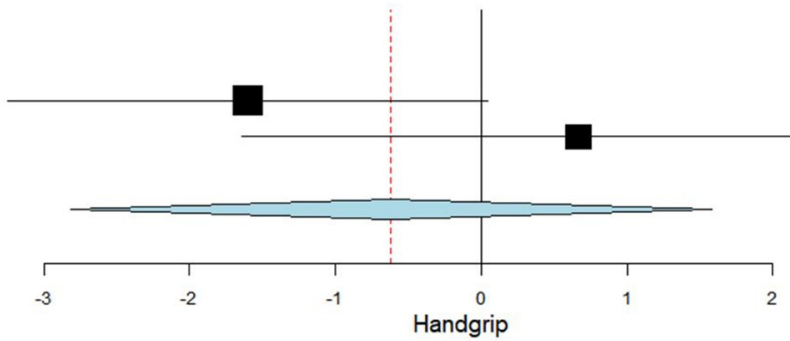

FIGURE 6 | Handgrip difference between baseline and post-BCAA supplementation.

Studies evaluated using the NHLBI quality assessment tool for observational cohort and cross-sectional studies were mainly rated as "fair" in four studies $(38,41,43,48)$ and one rated as "poor" (42). Generally, all included articles presented a clearly stated objective or research question. The study population was clearly specified and defined in three studies $(38,41,48)$. The time frame was considered sufficient to reasonably expect seeing an association between BCAA supplementation and changes in 
parameters in only one study (41). None of the five articles evaluated using this tool clearly stated that assessors were blinded to the exposure status of the participants. Only two studies assessed potential confounding variables and performed statistical adjustments for their impact $(38,48)$.

A total of three studies comparing baseline with postintervention values were evaluated using the NHLBI Quality Assessment Tool for Before-After (Pre-Post) Studies with No Control Group, with one article rated as "good" (44), one as "poor" (45), and one as "fair" (47). All three studies had a clear objective or research question, as well as clearly described eligibility criteria for the study population. All three articles used statistical methods to examine the outcome measure from before and after the intervention $(44,45,47)$. Only one study did not assess the outcome measure multiple times after the underwent intervention (45).

\section{DISCUSSION}

Recently, the interest in sarcopenia in liver cirrhosis has increased significantly. Several published reviews evaluated the effects of interventions, including BCAA supplementation, on sarcopenia in patients with cirrhosis $(11,50,51)$. However, none evaluated the effects of BCAA on anthropometric parameters assessing sarcopenia in this group population through a meta-analysis. To the best of our knowledge, this is the first systematic review and meta-analysis to evaluate the effects of supplementation with BCAAs on parameters assessing sarcopenia in liver cirrhosis. We included a total of eleven studies in our qualitative synthesis with a total population of 1,215, mainly Asian individuals, and to a lesser extent, Caucasians and Hispanics. They participated in four RCTs and seven observational studies. Furthermore, we included five studies in our quantitative synthesis, in which we demonstrated a significant improvement in SMI and MAMC parameters following BCAA supplementation, compared to nonBCAA group or baseline values, without significant improvement in handgrip and triceps subcutaneous fat. Although M-DXT was shown to slightly increase MAMC more than BCAA supplementation, no statistically significant difference was found between both groups. Moreover, handgrip was slightly better in subjects receiving BCAA supplementation compared to DXT, but without a statistically significant difference between both groups.

The new guidelines emphasize the importance of performing a functional evaluation and muscle mass quantification for evaluating sarcopenia (52). Anthropometric measures such as MAMC and triceps subcutaneous fat have been used and remain of significant importance in daily practice, reported to correlate with lean muscle mass and body fat, with an acceptable predictive value $(53,54)$. Nevertheless, measurement errors can occur due to lack of inter-observer agreement, as well as reduced accuracy in case of fluid overload (55). Several tools have been proposed to quantify muscle mass in clinical practice, including hand grip strength, assessed by recording the mean value of the dominant arm gripping a dynamometer in three consecutive measurements, and the chair stand test (CST), evaluated by counting the number of times the patient is able to rise fully to a standing position and subsequently sitting down in 30 seconds, without using their hands (56). Although hand grip strength was reported as an independent factor of mortality (57), on cross-sectional imaging, it was found to weakly correlated with muscle mass and quality (58). Currently, the skeletal muscle area (SMA) can be obtained using CT scan with a specific software (56). Afterwards, skeletal muscle index (SMI) in $\mathrm{cm}^{2} / \mathrm{m}^{2}$ can be obtained, being easily performed as the abdomen is evaluated to diagnose liver cirrhosis, while being able to discriminate between ascites and soft tissues.

Recently, researchers have attempted to find strategies that can help decrease the increased anabolic turnover rate of muscle that is age-related (59). Protein is made up of amino acids, possibly inducing a muscle protein anabolic response that is according to the availability of BCAAs, including leucine, isoleucine, and valine $(37,60)$. In elderly subjects, anabolic resistance and delayed absorption of amino acid absorption can be seen (61). Decreased muscle mass and function, as well as weaker muscle strength in the elderly have been associated with decreased BCAA levels (62). Moreover, aerobic exercise was found to contribute to the inductions of mitochondrial biogenesis and dynamics, mitochondrial metabolism restoration, as well as decreases the catabolic genes expression and increases muscle protein synthesis (63). Furthermore, an important strategy for preventing muscle wasting includes resistance exercise that was reported to strengthen muscle mass and function through stimulating muscle hypertrophy and improving muscle strength (64). Therefore, combining aerobic and resistance exercises can provide a greater benefit, providing a partial solution to sarcopenia. Accordingly, several studies reported that BCAA supplementation, including leucine, isoleucine, valine, or essential amino acids, in addition to aerobic and low-intensity resistance training can attenuate sarcopenia and stimulate muscle protein synthesis, even in the bedrest confined elderly subjects (37).

We believe that our results need to be further discussed. Firstly, most involved participants were from Asia, with a limited number of Caucasians and Hispanics. Moreover, ethnicities such as African Americans were not included in any of the studies evaluating parameters of sarcopenia in patients with cirrhosis. Therefore, the obtained results cannot be generalized to other ethnicities that have not been evaluated yet in the currently published evidence.

In our meta-analysis, we demonstrated that several parameters, including SMI and MAMC were improved following BCAA supplementation. Nevertheless, handgrip and triceps subcutaneous fat did not improve significantly. Although current evidence demonstrated that supplementation with BCAAs such as leucine, valine, and isoleucine could ameliorate protein synthesis, lipid, and glucose metabolism, as well as insulin resistance and hepatocyte proliferation, in addition, reduce oxidative stress in hepatocytes in liver cirrhosis, several published studies reported no significant improvement in muscle strength or mass post-intervention with BCAA supplementation $(51,65)$. This can be partially explained by the short intervention duration and small sample size (40, 66-68), or a specific subgroup of LC with albumin $\leq 3.5 \mathrm{~g} / \mathrm{dL}(44,45)$. Furthermore, administration timing, dose, and nutritional 
education regarding BCAA supplementation are also considered essential factors that might lead to suboptimal results if not properly performed. The present systematic review and metaanalysis evaluates parameters used to assess sarcopenia and did not assess sarcopenia as its presence or absence. However, it is important to assess the effects of BCAAs on these parameters, used to evaluate sarcopenia in patients with liver cirrhosis. Any findings in this respect can help gather evidence on how to treat sarcopenia as well.

According to the quality assessment of included studies in our systematic review and meta-analysis, most evaluated studies using both NHLBI quality assessment tools were rated as "poor" and "fair," while only one study was evaluated as "good." Moreover, half of the included RCTs presented an unclear risk of allocation concealment bias, in addition to unclear risk of reporting bias and other possible sources of bias in all included RCTs. Another point to consider is that several studies did not perform statistical adjustments for potential confounding variables. Therefore, the obtained results from studies with poor and fair methodological quality should be cautiously interpreted.

Most included studies were of observational design and not interventional. Therefore, more interventional studies are required in order to confirm the possible causality between BCAA supplementation and improvements in parameters assessing sarcopenia in patients with cirrhosis. Furthermore, we were not able to perform subgroup analysis evaluating variables according to the etiology of liver cirrhosis or administrated BCAA supplementation due to limited available data.

Our systematic review and meta-analysis has several limitations that need to be addressed. Multiple included studies in this review are of observational design. Therefore, causality between BCAA supplementation and improvement or worsening of sarcopenia assessed parameters in cirrhosis cannot be confirmed or negated according to these studies. Although several parameters assessing sarcopenia, including muscle mass, function, and strength evaluation, were conducted in the included studies in our review, most studies had a different grouping of the involved participants or interventions performed, leading to a very limited number of studies that were possibly included in our quantitative synthesis. Furthermore, due to a limited number of published articles assessing several parameters used to assess sarcopenia in liver cirrhosis patients receiving BCAA supplements, we could only assess two studies for each association. The pre-post comparisons encountered in some meta-analyses are subject to possible biases, being less desirable to classic between-arms comparisons. Short intervention intervals and small sample sizes can also lead to suboptimal results. Accordingly, future research involving larger populations with longer interventional intervals involving BCAA supplementation is deemed necessary. Due to possible methodological flaws in included studies, results should be interpreted with caution.

Nevertheless, our systematic review and meta-analysis also has several important strengths. We believe that this topic is of important clinical significance due to the increased prevalence of liver cirrhosis and secondary sarcopenia, leading to more complications and higher morbidity and mortality rates. In this review, we point out several problems in current studies that should be remediated in future studies. We also performed a comprehensive search in several electronic databases while metaanalytically summarizing the current literature regarding this topic in a non-biased manner. To the best of our knowledge, this is the first systematic review and meta-analysis to assess the effects of BCAA supplementation on sarcopenia evaluated parameters in liver cirrhosis.

\section{CONCLUSIONS AND FUTURE DIRECTIONS}

Improvements in several parameters used to assess sarcopenia in liver cirrhosis patients, including skeletal muscle index, mid-arm muscle circumference, were seen following BCAA supplementation. However, no improvements were seen in handgrip and triceps subcutaneous fat. Nonetheless, due to the imperfect methodological quality of the evaluated articles, interpretation of the obtained results should be performed with caution.

Future interventional studies, mainly better methodologically conducted RCTs, with larger sample sizes and longer interventional intervals evaluating the effects of BCAA supplementation on parameters used to assess sarcopenia in liver cirrhosis patients from different ethnicities, remains necessary. Possible improvements in quality of life, survival, mobility, and cardiopulmonary performance, in addition to reduced infection rates and favorable metabolic outcomes in liver cirrhosis patients with sarcopenia, can be obtained if future RCTs confirm our reported findings. Moreover, a clear consensus defining sarcopenia in liver cirrhosis patients is required.

\section{DATA AVAILABILITY STATEMENT}

The original contributions presented in the study are included in the article/Supplementary Material, further inquiries can be directed to the corresponding author.

\section{AUTHOR CONTRIBUTIONS}

D-CL and DD had the idea of the manuscript and made substantial contributions to the conception and critically revised the manuscript for important intellectual content. AI and D-CL independently applied the search strategy, performed the study selection, and risk of bias assessment. $\mathrm{CB}$ and $\mathrm{AF}$ performed the data extraction. AI drafted the manuscript. D-CL, CB, AF, S-LP, and DD contributed to the writing of the manuscript. All authors revised the final manuscript and approved the final version.

\section{SUPPLEMENTARY MATERIAL}

The Supplementary Material for this article can be found online at: https://www.frontiersin.org/articles/10.3389/fnut.2022. 749969/full\#supplementary-material 


\section{REFERENCES}

1. Rosenberg IH. Summary comments. Am J Clin Nutr. (1989) 50:12313. doi: 10.1093/ajcn/50.5.1231

2. Rosenberg IH. Sarcopenia: origins and clinical relevance. J. Nutr. (1997) 127:990s-1s. doi: 10.1093/jn/127.5.990S

3. Cruz-Jentoft AJ, Bahat G, Bauer J, Boirie Y, Bruyère $O$, Cederholm $T$, et al. Sarcopenia: revised European consensus on definition and diagnosis. Age Ageing. (2019) 48:16-31. doi: 10.1093/ageing/afy169

4. Chen LK, Woo J, Assantachai P, Auyeung TW, Chou MY, Iijima K, et al. Asian working group for sarcopenia: 2019 consensus update on sarcopenia diagnosis and treatment. J. Am. Med. Dir. Assoc. (2020) 21:3007.e302. doi: 10.1016/j.jamda.2019.12.012

5. Dasarathy S, Merli M. Sarcopenia from mechanism to diagnosis and treatment in liver disease. J Hepatol. (2016) 65:1232-44. doi: 10.1016/j.jhep.2016.07.040

6. Jindal A, Jagdish RK. Sarcopenia: Ammonia metabolism and hepatic encephalopathy. Clin Mol Hepatol. (2019) 25:2709. doi: 10.3350/cmh.2019.0015

7. Son SW, Song DS, Chang UI, Yang JM. Definition of Sarcopenia in Chronic Liver Disease. Life (Basel, Switzerland). (2021) 11:349. doi: 10.3390/life11040349

8. Torre-Delgadillo A. [Complications of cirrhosis: encephalopathy, nutritional status, and ascites]. Rev Gastroenterol Mex. (2013) 78(Suppl. 1):1035. doi: 10.1016/j.rgmx.2013.07.001

9. Ruiz-Margáin A, Macías-Rodríguez RU, Duarte-Rojo A, Ríos-Torres SL, Espinosa-Cuevas Á, Torre A. Malnutrition assessed through phase angle and its relation to prognosis in patients with compensated liver cirrhosis: a prospective cohort study. Dig Liver Dis. (2015) 47:309-14. doi: 10.1016/j.dld.2014.12.015

10. Fielding RA, Vellas B, Evans WJ, Bhasin S, Morley JE, Newman AB, et al. Sarcopenia: an undiagnosed condition in older adults. Current consensus definition: prevalence, etiology, and consequences International working group on sarcopenia. J Am Med Dir Assoc. (2011) 12:24956. doi: 10.1016/j.jamda.2011.01.003

11. Dhaliwal A, Armstrong MJ. Sarcopenia in cirrhosis: a practical overview. Clin Med. (2020) 20:489-92. doi: 10.7861/clinmed.2020-0089

12. Dasarathy S. Consilience in sarcopenia of cirrhosis. J Cachexia Sarcopenia Muscle. (2012) 3:225-37. doi: 10.1007/s13539-012-0069-3

13. Periyalwar P, Dasarathy S. Malnutrition in cirrhosis: contribution and consequences of sarcopenia on metabolic and clinical responses. Clin Liver Dis. (2012) 16:95-131. doi: 10.1016/j.cld.2011.12.009

14. Koretz RL, Avenell A, Lipman TO. Nutritional support for liver disease. Cochrane Database Syst Rev. (2012) 2012:Cd008344. doi: 10.1002/14651858.CD008344.pub2

15. Eghtesad S, Poustchi H, Malekzadeh R. Malnutrition in liver cirrhosis:the influence of protein and sodium. Middle East J Dig Dis. (2013) 5:65-75.

16. Katayama K, Saito M, Kawaguchi T, Endo R, Sawara K, Nishiguchi $S$, et al. Effect of zinc on liver cirrhosis with hyperammonemia: a preliminary randomized, placebo-controlled double-blind trial. Nutrition. (2014) 30:1409-14. doi: 10.1016/j.nut.2014.04.018

17. Iob V, Coon WW, Sloan M. Free amino acids in liver, plasma, and muscle of patients with cirrhosis of the liver. J Surg Res. (1967) 7:413. doi: 10.1016/0022-4804(67)90008-X

18. Kawaguchi T, Taniguchi E, Sata M. Effects of oral branched-chain amino acids on hepatic encephalopathy and outcome in patients with liver cirrhosis. Nutr Clin Pract. (2013) 28:580-8. doi: 10.1177/08845336134 96432

19. Muto Y, Sato S, Watanabe A, Moriwaki H, Suzuki K, Kato A, et al. Effects of oral branched-chain amino acid granules on event-free survival in patients with liver cirrhosis. Clin Gastroenterol Hepatol. (2005) 3:70513. doi: 10.1016/S1542-3565(05)00017-0

20. Charlton M. Branched-chain amino acid enriched supplements as therapy for liver disease. J. Nutr. (2006) 136:295s-8s. doi: 10.1093/jn/136.1.295S

21. Kawaguchi T, Izumi N, Charlton MR, Sata M. Branched-chain amino acids as pharmacological nutrients in chronic liver disease. Hepatology. (2011) 54:1063-70. doi: 10.1002/hep.24412

22. Nishitani S, Takehana K, Fujitani S, Sonaka I. Branchedchain amino acids improve glucose metabolism in rats with liver cirrhosis. Am J Physiol Gastrointest Liver Physiol.

(2005) 288:G1292-1300. doi: 10.1152/ajpgi.00510.2003

23. Hayaishi S, Chung H, Kudo M, Ishikawa E, Takita M, Ueda T, et al. Oral branched-chain amino acid granules reduce the incidence of hepatocellular carcinoma and improve event-free survival in patients with liver cirrhosis. Dig Dis. (2011) 29:326-32. doi: 10.1159/000327571

24. Nishikawa $\mathrm{H}$, Osaki $\mathrm{Y}$, Inuzuka $\mathrm{T}$, Takeda $\mathrm{H}$, Nakajima J, Matsuda F, et al. Branched-chain amino acid treatment before transcatheter arterial chemoembolization for hepatocellular carcinoma. World J Gastroenterol. (2012) 18:1379-84. doi: 10.3748/wjg.v18.i12.1379

25. Ichikawa $K$, Okabayashi $T$, Maeda $H$, Namikawa $T$, Iiyama $T$, Sugimoto $\mathrm{T}$, et al. Oral supplementation of branched-chain amino acids reduces early recurrence after hepatic resection in patients with hepatocellular carcinoma: a prospective study. Surg Today. (2013) 43:720-6. doi: 10.1007/s00595-012-0288-4

26. Page MJ, Mckenzie JE, Bossuyt PM, Boutron I, Hoffmann TC, Mulrow CD, et al. The PRISMA 2020 statement: an updated guideline for reporting systematic reviews. BMJ. (2021) 372:n71. doi: 10.1136/bmj.n71

27. Higgins J, Sally G. Cochrane Handbook for Systematic Reviews of Interventions. (2011). Available online at: http://handbook-5-1.cochrane.org/ (accessed 15 July 2021).

28. Health NIO. Quality Assessment Tool for Observational Cohort and CrossSectional Studies. (2014). Available online at: https://www.nhlbi.nih.gov/ health-topics/study-quality-assessment-tools (accessed 23 July 2021].

29. Viechtbauer W. Conducting meta-analyses in $\mathrm{R}$ with the metafor Package. $J$ Stat Softw. (2010) 36:48. doi: 10.18637/jss.v036.i03

30. Wallace BC, Dahabreh IJ, Trikalinos TA, Lau J, Trow P, Schmid CH. Closing the gap between methodologists and end-users: $\mathrm{R}$ as a computational backend. https://www.researchgate.net/journal/Journal-of-Statistical-Software1548-7660J Stat Softw. (2012) 49:15. doi: 10.18637/jss.v049.i05

31. Higgins Jpt GSE. Cochrane Handbook for Systematic Reviews of Interventions Version 5.1.0. (2011). London: The Cochrane Collaboration.

32. Wan X, Wang W, Liu J, Tong T. Estimating the sample mean and standard deviation from the sample size, median, range and/or interquartile range. BMC Med Res Methodol. (2014) 14:135. doi: 10.1186/1471-2288-14-135

33. Román E, Torrades MT, Nadal MJ, Cárdenas G, Nieto JC, Vidal S, et al. Randomized pilot study: effects of an exercise programme and leucine supplementation in patients with cirrhosis. Dig Dis Sci. (2014) 59:196675. doi: 10.1007/s10620-014-3086-6

34. Hiraoka A, Aibiki T, Okudaira T, Toshimori A, Kawamura T, Nakahara H, et al. Muscle atrophy as pre-sarcopenia in Japanese patients with chronic liver disease: computed tomography is useful for evaluation. J Gastroenterol. (2015) 50:1206-13. doi: 10.1007/s00535-015-1068-x

35. Conde MH, Llop E, Tormo B, Perelló C, López-Gómez M, Guerra JA, et al. Supplementation with branched-chain amino acids improves muscle mass of cirrhotic patients with sarcopenia. J Hepatol. (2020) 73:S378. doi: 10.1016/S0168-8278(20)30627-9

36. Sano A, Tsuge S, Kakazu E, Iwata T, Ninomiya M, Tsuruoka M, et al. Plasma free amino acids are associated with sarcopenia in the course of hepatocellular carcinoma recurrence. Nutrition. (2021) 84. doi: 10.1016/j.nut.2020.1 11007

37. Ko C-H, Wu S-J, Wang S-T, Chang Y-F, Chang C-S, Kuan T-S, et al. Effects of enriched branched-chain amino acid supplementation on sarcopenia. Aging (Milano). (2020) 12:15091-103. doi: 10.18632/aging.103576

38. Hanai T, Shiraki M, Watanabe S, Kochi T, Imai K, Suetsugu A, et al. Sarcopenia predicts minimal hepatic encephalopathy in patients with liver cirrhosis. Hepatol Res. (2017) 47:1359-67. doi: 10.1111/hepr.12873

39. Marchesini G, Bianchi G, Merli M, Amodio P, Panella C, Loguercio C, et al. Nutritional supplementation with branched-chain amino acids in advanced cirrhosis: a double-blind, randomized trial. Gastroenterology. (2003) 124:1792-801. doi: 10.1016/S0016-5085(03)00323-8

40. Les I, Doval E, García-Martínez R, Planas M, Cárdenas G, Gómez P, et al. Effects of branched-chain amino acids supplementation in patients with cirrhosis and a previous episode of hepatic encephalopathy: a randomized study. Am J Gastroenterol. (2011) 106:1081-8. doi: 10.1038/ajg.2011.9

41. Hanai T, Shiraki M, Nishimura K, Ohnishi S, Imai K, Suetsugu A, et al. Sarcopenia impairs prognosis of patients with liver cirrhosis. Nutrition. (2015) 31:193-9. doi: 10.1016/j.nut.2014.07.005 
42. Tsien C, Davuluri G, Singh D, Allawy A, Ten Have GAM, Thapaliya S, et al. Metabolic and molecular responses to leucine-enriched branched chain amino acid supplementation in the skeletal muscle of alcoholic cirrhosis. Hepatology. (2015) 61:2018-29. doi: 10.1002/hep.27717

43. Hiraoka A, Michitaka K, Kiguchi D, Izumoto H, Ueki H, Kaneto M, et al. Efficacy of branched-chain amino acid supplementation and walking exercise for preventing sarcopenia in patients with liver cirrhosis. Eur J Gastroenterol Hepatol. (2017) 29:1416-23. doi: 10.1097/MEG.0000000000000986

44. Uojima H, Sakurai S, Hidaka H, Kinbara T, Sung JH, Ichita C, et al. Effect of branched-chain amino acid supplements on muscle strength and muscle mass in patients with liver cirrhosis. Eur J Gastroenterol Hepatol. (2017) 29:1402-7. doi: 10.1097/MEG.0000000000000968

45. Kitajima Y, Takahashi H, Akiyama T, Murayama K, Iwane S, Kuwashiro $\mathrm{T}$, et al. Supplementation with branched-chain amino acids ameliorates hypoalbuminemia, prevents sarcopenia, and reduces fat accumulation in the skeletal muscles of patients with liver cirrhosis. J Gastroenterol. (2018) 53:427-37. doi: 10.1007/s00535-017-1370-x

46. Ruiz-Margáin A, Macías-Rodríguez RU, Ríos-Torres SL, Román-Calleja BM, Méndez-Guerrero O, Rodríguez-Córdova P, et al. Effect of a high-protein, high-fiber diet plus supplementation with branched-chain amino acids on the nutritional status of patients with cirrhosis. Rev Gastroenterol Mex. (2018) 83:9-15. doi: 10.1016/j.rgmxen.2017.02.005

47. Hiraoka A, Kiguchi D, Ninomiya T, Hirooka M, Abe M, Matsuura $B$, et al. Can l-carnitine supplementation and exercise improve muscle complications in patients with liver cirrhosis who receive branched-chain amino acid supplementation? Eur. J Gastroenterol Hepatol. (2019) 31:87884. doi: 10.1097/MEG.0000000000001368

48. Hanai T, Shiraki M, Imai K, Suetsugu A, Takai K, Shimizu M. Late evening snack with branched-chain amino acids supplementation improves survival in patients with cirrhosis. J Clin Med. (2020) 9:1013. doi: 10.3390/jcm9041013

49. Okubo T, Atsukawa M, Tsubota A, Ono H, Kawano T, Yoshida Y, et al. Effect of vitamin d supplementation on skeletal muscle volume and strength in patients with decompensated liver cirrhosis undergoing branched chain amino acids supplementation: a prospective, randomized, controlled pilot trial. Nutrients. (2021) 13:1874. doi: 10.3390/nu13061874

50. Ponziani FR, Gasbarrini A. Sarcopenia in patients with advanced liver disease. Curr Protein Pept Sci. (2018) 19:68191. doi: 10.2174/1389203718666170428121647

51. Naseer M, Turse EP, Syed A, Dailey FE, Zatreh M, Tahan V. Interventions to improve sarcopenia in cirrhosis: a systematic review. World J Clin Cases. (2019) 7:156-70. doi: 10.12998/wjcc.v7.i2.156

52. Plauth M, Bernal W, Dasarathy S, Merli M, Plank LD, Schütz T, et al. ESPEN guideline on clinical nutrition in liver disease. Clin Nutr. (2019) 38:485-521. doi: 10.1016/j.clnu.2018.12.022

53. Caregaro L, Alberino F, Amodio P, Merkel C, Bolognesi M, Angeli P, et al. Malnutrition in alcoholic and virus-related cirrhosis. Am J Clin Nutr. (1996) 63:602-9. doi: 10.1093/ajcn/63.4.602

54. Alberino F, Gatta A, Amodio P, Merkel C, Di Pascoli L, Boffo G, et al. Nutrition and survival in patients with liver cirrhosis. Nutrition. (2001) 17:445-50. doi: 10.1016/S0899-9007(01)00521-4

55. Ulijaszek SJ, Kerr DA. Anthropometric measurement error and the assessment of nutritional status. Br J Nutr. (1999) 82:165-77. doi: 10.1017/S0007114599001348

56. Buchard B, Boirie Y, Cassagnes L, Lamblin G, Coilly A, Abergel A. Assessment of malnutrition, sarcopenia and frailty in patients with cirrhosis: which tools should we use in clinical practice? Nutrients. (2020) 12:186. doi: 10.3390/nu12010186

57. Sinclair M, Chapman B, Hoermann R, Angus PW, Testro A, Scodellaro T, et al. Handgrip strength adds more prognostic value to the model for end-stage liver disease score than imaging-based measures of muscle mass in men with cirrhosis. Liver Transpl. (2019) 25:1480-7. doi: 10.1002/lt.25598
58. Wang CW, Feng S, Covinsky KE, Hayssen H, Zhou LQ, Yeh BM, et al. A comparison of muscle function, mass, and quality in liver transplant candidates: results from the functional assessment in liver transplantation study. Transplantation. (2016) 100:1692-8. doi: 10.1097/TP.0000000000001232

59. Dardevet D, Rémond D, Peyron MA, Papet I, Savary-Auzeloux I, Mosoni L. Muscle wasting and resistance of muscle anabolism: the "anabolic threshold concept" for adapted nutritional strategies during sarcopenia. ScientificWorldJournal. (2012) 2012:269531. doi: 10.1100/2012/ 269531

60. Walker DK, Dickinson JM, Timmerman KL, Drummond MJ, Reidy PT, Fry CS, et al. Exercise, amino acids, and aging in the control of human muscle protein synthesis. Med Sci Sports Exerc. (2011) 43:224958. doi: 10.1249/MSS.0b013e318223b037

61. Burd NA, Gorissen SH, Van Loon LJ. Anabolic resistance of muscle protein synthesis with aging. Exerc Sport Sci Rev. (2013) 41:16973. doi: 10.1097/JES.0b013e318292f3d5

62. Ter Borg S, Luiking YC, Van Helvoort A, Boirie Y, Schols J, De Groot C. Low levels of branched chain amino acids, eicosapentaenoic acid and micronutrients are associated with low muscle mass, strength and function in community-dwelling older adults. J Nutr Health Aging. (2019) 23:2734. doi: 10.1007/s12603-018-1108-3

63. Yoo S-Z, No M-H, Heo J-W, Park D-H, Kang J-H, Kim SH, et al. Role of exercise in age-related sarcopenia. J Exerc Rehabil. (2018) 14:5518. doi: 10.12965/jer.1836268.134

64. Johnston AP, De Lisio M, Parise G. Resistance training, sarcopenia, and the mitochondrial theory of aging. Appl Physiol Nutr Metab. (2008) 33:1919. doi: 10.1139/H07-141

65. Toshikuni N, Arisawa T, Tsutsumi M. Nutrition and exercise in the management of liver cirrhosis. World J Gastroenterol. (2014) 20:728697. doi: 10.3748/wjg.v20.i23.7286

66. Yamanaka-Okumura H, Nakamura T, Takeuchi H, Miyake H, Katayama $\mathrm{T}$, Arai $\mathrm{H}$, et al. Effect of late evening snack with rice ball on energy metabolism in liver cirrhosis. Eur J Clin Nutr. (2006) 60:106772. doi: 10.1038/sj.ejen. 1602420

67. Nakaya Y, Okita K, Suzuki K, Moriwaki H, Kato A, Miwa Y, et al. BCAAenriched snack improves nutritional state of cirrhosis. Nutrition. (2007) 23:113-20. doi: 10.1016/j.nut.2006.10.008

68. Dupont B, Dao T, Joubert C, Dupont-Lucas C, Gloro R, Nguyen-Khac E, et al. Randomised clinical trial: enteral nutrition does not improve the long-term outcome of alcoholic cirrhotic patients with jaundice. Aliment Pharmacol Ther. (2012) 35:1166-74. doi: 10.1111/j.1365-2036.2012.05075.x

Conflict of Interest: The authors declare that the research was conducted in the absence of any commercial or financial relationships that could be construed as a potential conflict of interest.

Publisher's Note: All claims expressed in this article are solely those of the authors and do not necessarily represent those of their affiliated organizations, or those of the publisher, the editors and the reviewers. Any product that may be evaluated in this article, or claim that may be made by its manufacturer, is not guaranteed or endorsed by the publisher.

Copyright (C) 2022 Ismaiel, Bucsa, Farcas, Leucuta, Popa and Dumitrascu. This is an open-access article distributed under the terms of the Creative Commons Attribution License (CC BY). The use, distribution or reproduction in other forums is permitted, provided the original author(s) and the copyright owner(s) are credited and that the original publication in this journal is cited, in accordance with accepted academic practice. No use, distribution or reproduction is permitted which does not comply with these terms. 